\title{
Integrated adaptive optics system for small telescopes
}

\author{
Mikhail Loktev $^{1,2}$, Gleb Vdovin ${ }^{1,2}$ and Oleg Soloviev ${ }^{1}$ \\ ${ }^{1}$ Flexible Optical B.V. (OKO Technologies), \\ Röntgenweg 1, 2624 BD, Delft, the Netherlands \\ ${ }^{2}$ Electronic Instrumentation Laboratory, Delft University of Technology, \\ Mekelweg 4, 2628 CD, Delft, the Netherlands
}

\begin{abstract}
We describe a compact integrated module implementing a low-cost adaptive optics system. It is targeted as a correction system for small telescopes with primary mirror diameter up to $1 \mathrm{~m}$, operating on a natural guide star with magnitude at least 4 (for a $25 \mathrm{~cm}$ telescope). It is supposed to provide stable diffraction-limited imaging of stars, double stars, planets and artificial bodies in various seeing conditions. Besides, it allows correcting for static aberrations of the telescope, observer's ocular aberrations and fine adjustment of focus. The first on-sky tests have demonstrated closed-loop operation with correction for aberrations.
\end{abstract}

Keywords: Amateur astronomy, adaptive optics, deformable mirror, wavefront corrector.

\section{CONCEPT OF A LOW-COST AO SYSTEM}

Low-cost adaptive optics is the bet of technology-pushers. It is widely believed that as soon as adaptive optics is inexpensive, many useful and profitable applications will arrive. One of these potential applications is in astronomy. The modern high-end astronomy is already strongly dependent on the adaptive optics, however the low-end astronomy with small telescopes and low budgets is believed to stay free of adaptive optics simply because the amateurs cannot pay for using the AO. Moreover, it is more difficult technically to build an AO system for a light-starved small telescope, and the usefulness of such a system is frequently questionable.

On the other hand, a low-cost AO system would be quite beneficial even in statical case, as many amateurs do not have any good permanent location for astronomic observations and use portable systems exposed to static aberrations. ${ }^{1}$ Dynamically, the AO system could provide a certain improvement in the performance compared to existing commercial systems for amateur telescopes that only partly correct for tip-tilt. ${ }^{2,3}$ The real AO should allow for stable imaging of bright objects such as stars, double stars, planets and other bodies.

Another advantage of using such a system is that it can correct for the aberrations of the telescope itself, improving the quality of optics, for instance making the cooling period of the mirror of a portable telescope also available for observations. In some cases, the correction for the static aberration can be done on a bright star only once, and then the system can be used in the corrected mode.

Preliminary technical requirements for such a system (based on a very useful discussion in sci.astro.amateur newsgroup) are as follows:

- To be mounted in 1.25 inch ocular socket.

- To be used with telescopes with diameter in the range of $25 \mathrm{~cm}$ to $1 \mathrm{~m}$.

- To be implemented as an afocal mirror system, transparent in the visible and near IR and fully operational even with AO switched off. To achieve this, we'll use a system with a field of a couple of $\mathrm{mm}$ (in the primary focus) for a focal ratio of $1 / 10$. The field and $\mathrm{F} / \#$ are compromised to reduce the complexity of the optics, but the field will be limited anyway by the anisoplanatism of the AO and the F/\# must be small for a high-resolution imaging.

Further author information:

M.L. (e-mail): misha@okotech.com, G.V. (e-mail): gleb@okotech.com, O.S. (e-mail): oleg@okotech.com

Adaptive Optics Systems, edited by Norbert Hubin, Claire E. Max, Peter L. Wizinowich,

Proc. of SPIE Vol. 7015, 70153K, (2008)

0277-786X/08/\$18 doi: $10.1117 / 12.787893$

Proc. of SPIE Vol. 7015 70153K-1 
- To have al least 19 degrees of freedom (depending on the seeing can be good to correct up to $~ 13$ Zernike terms to about $10 \%$ of the uncorrected value).

- To operate on a natural star with magnitude of at least 4 (with a $25 \mathrm{~cm}$ telescope), using $50 \%$ of light for running the $\mathrm{AO}$ and $50 \%$ for registration.

- To be easy in setting up and running. To use two cables connecting the system with the deformable mirror controller and Shack-Hartmann wavefront sensor, both operated by a dedicated laptop PC.

- The total weight of the optical correction unit mounted to the telescope should not exceed $2 \mathrm{~kg}$. The mirror controller should weight about $1 \mathrm{~kg}$ including power supply, with some extra for cables and laptop.

- In case of serial production, the target price is about 5 thousand Euros for the complete setup without computer.

The idea of a low-end AO for an amateur is complementary to the future giant AO projects. Such a system will certainly have at least some market, bringing the low-cost AO to students and amateur astronomers and making the $\mathrm{AO}$ foundations in the professional astronomy even more solid. When used in non-astronomical fields, the newly developed portable AO module will facilitate integration of the AO into existing systems, saving the development time and money.

\section{OPTICAL SYSTEM}

The optical correction unit is built using afocal Offner configuration with two spherical mirrors. It has two functions:

- It images the input pupil onto the deformable mirror and

- it translates the position of the primary focus without changing the geometrical beam parameters (but correcting for the aberrations).

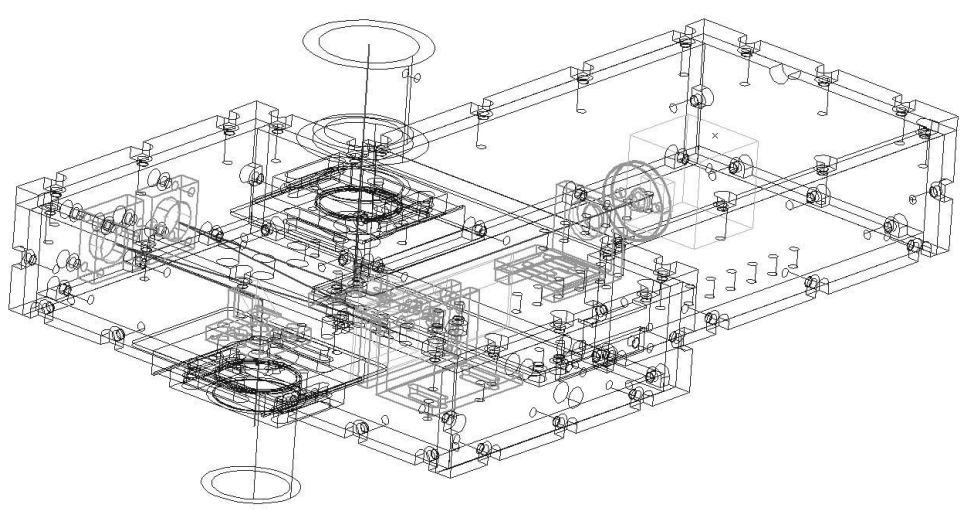

Figure 1. CAD drawing of the portable compact AO module.

The correction for aberrations is performed by a specially designed micromachined membrane deformable mirror $(\mathrm{MMDM}){ }^{4}$

The wavefront sensing part consists of a low-cost CCD camera with a USB interface. The camera is fed by a $50 \%$ of the available light through a beam splitter. The Hartmann-Shack sensor operates with 45 subapertures. The system is controlled by the FrontSurfer software package developed by OKO Technologies.

The CAD drawing of the first prototype of the optical system is shown in Figure 1. 


\section{EXPERIMENTAL RESULTS}

\subsection{Breadboard tests}

The first prototype of the system with total dimensions of 188x291x145 mm was assembled and tested at OKO Technologies. The optical correction unit mounted on $25 \mathrm{~cm}$ a telescope is shown in Figure 2.

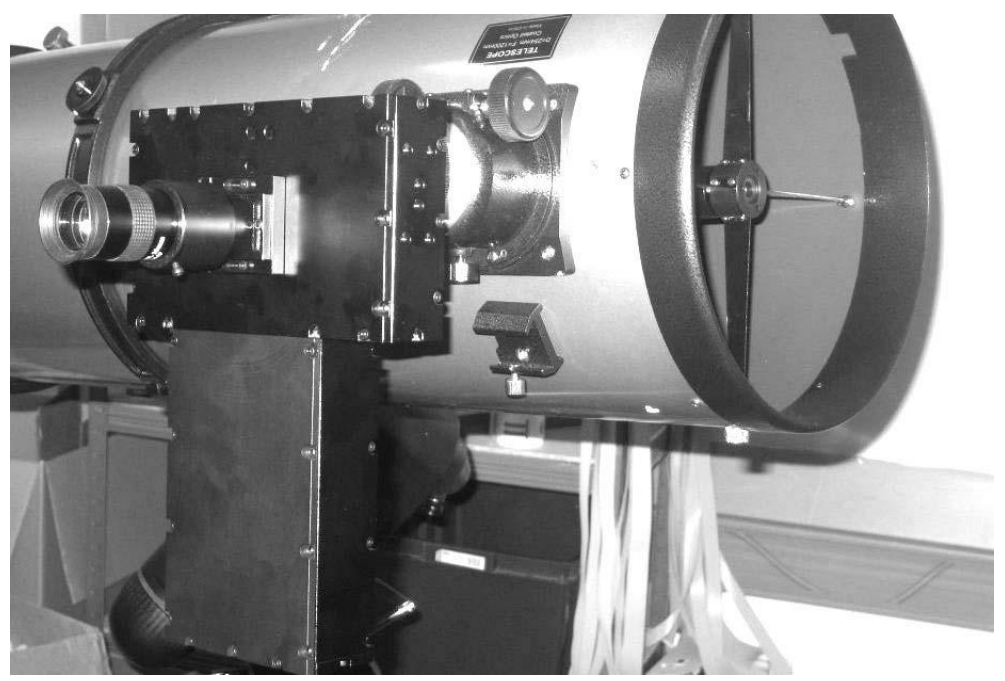

Figure 2. Compact AO module mounted on a $25 \mathrm{~cm}$ telescope.
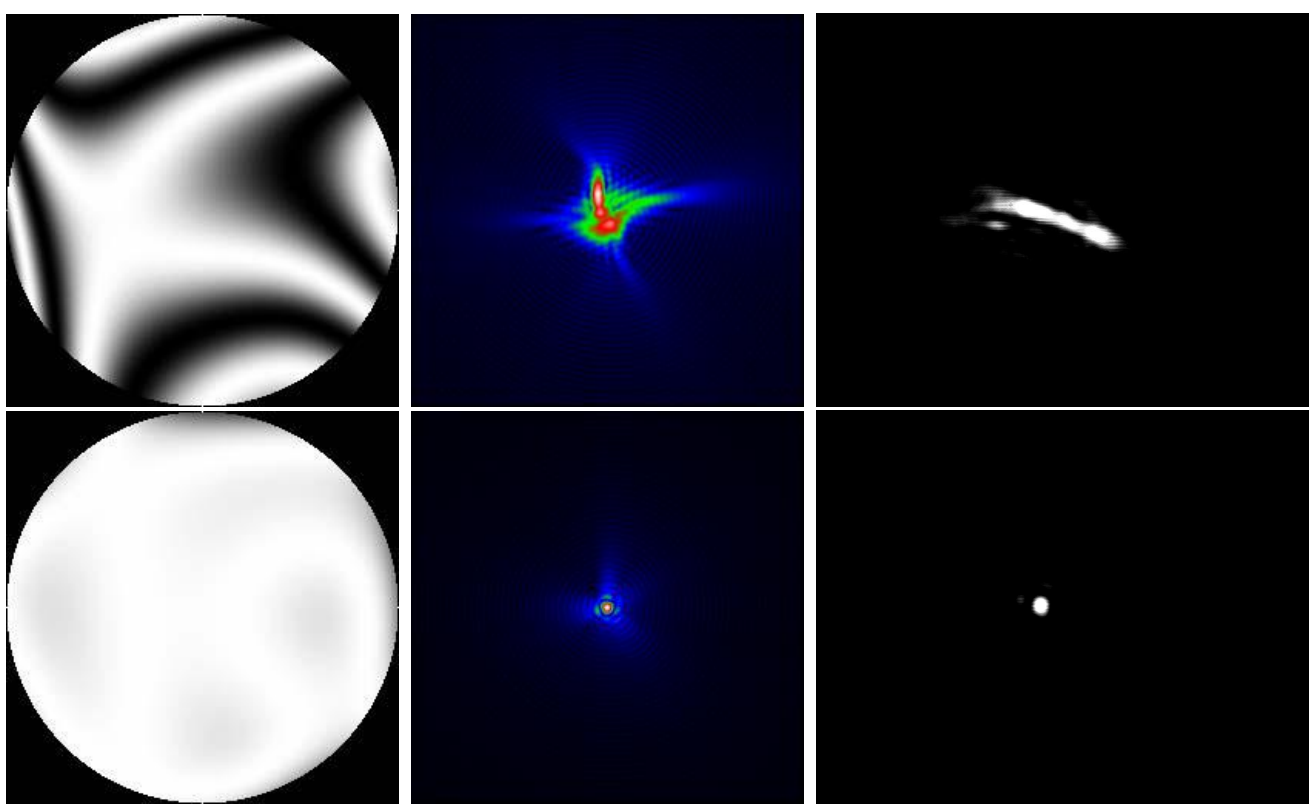

Figure 3. Interferogram of the intial aberration of the assembled AOS, model far field, measured far field (top row left to right); interferogram of the corrected aberration of the assembled AOS, model far field, measured far field (bottom row, left to right)

The first test of the AO system was performed with a laser source. A clean aberration-free collimated light beam was focused into the primary focus. Exactly as it was predicted by the model, the system has shown some amount of astigmatism caused by the off-axis operation in the Offner setup. This astigmatism - see Figure 3 is almost completely compensated by the closed-loop operation of the system. 
A dynamic correction test was done with the dynamic aberration introduced by a rotating disk aberrator. The system demonstrated satisfactory correction with frame frequency of the order of $45 \mathrm{~Hz}$. This frequency can be significantly increased by using a more powerful laptop.

\subsection{Testing on a 25-cm Newtonian telescope}

In another test, the system was mounted on a $25 \mathrm{~cm}$ Newtonian telescope with $1.2 \mathrm{~m}$ focus. We used a $2 \mathrm{x}$ Barlow lens to adapt the focal number of the telescope to the range acceptable for the AO system. We corrected the image of a LED placed at a distance of about $50 \mathrm{~m}$ away - which also served as a guide star for running the adaptive optics.

In August 2007, we tested the system on real sky objects: Altair (apparent magnitude of 0.75) and Sadr (magnitude of 2.21). In both cases, the object also served as a guide star for running the adaptive optics. The wavefront sensor was operated with software-generated reference, that allowed for fast dynamic correction combined with manual control of major low-order Zernike terms.

With the AO correction off, the image was strongly aberrated, mainly due to the astigmatism of the AO system; static aberrations of the telescope and the dynamic aberrations due to the atmospheric turbulence were found less significant in these tests. With the AO correction on, the static aberrations were compensated which resulted in the subjective image quality similar or better than those without the AO correction. FrontSurfer controls were used for fine adjustment of the defocus and low-order aberrations. We found that the AO allows for a very fine focus control, much finer than with the mechanical adjustments of the telescope. Since the FrontSurfer software allows for precise control of low-order Zernike terms, we found it to be a very efficient tool for subjective corrections for the static aberrations of the telescope and even of the observer's eye - in a way similar to the described in. ${ }^{5}$

\subsection{Testing on a 36-cm Schmidt-Cassegrainian telescope}

In order to check how the system works on a larger telescope and collect more data on the aberrations affecting on-sky observations, we tested the system on a $36 \mathrm{~cm}$ Schmidt-Cassegrainian telescope with $4 \mathrm{~m}$ focus. We performed a series of wavefront measurements with Mars used as a guide star; the wavefront sensor operated at a frame rate of $45 \mathrm{~Hz}$. The results are presented in Figures 4-6.
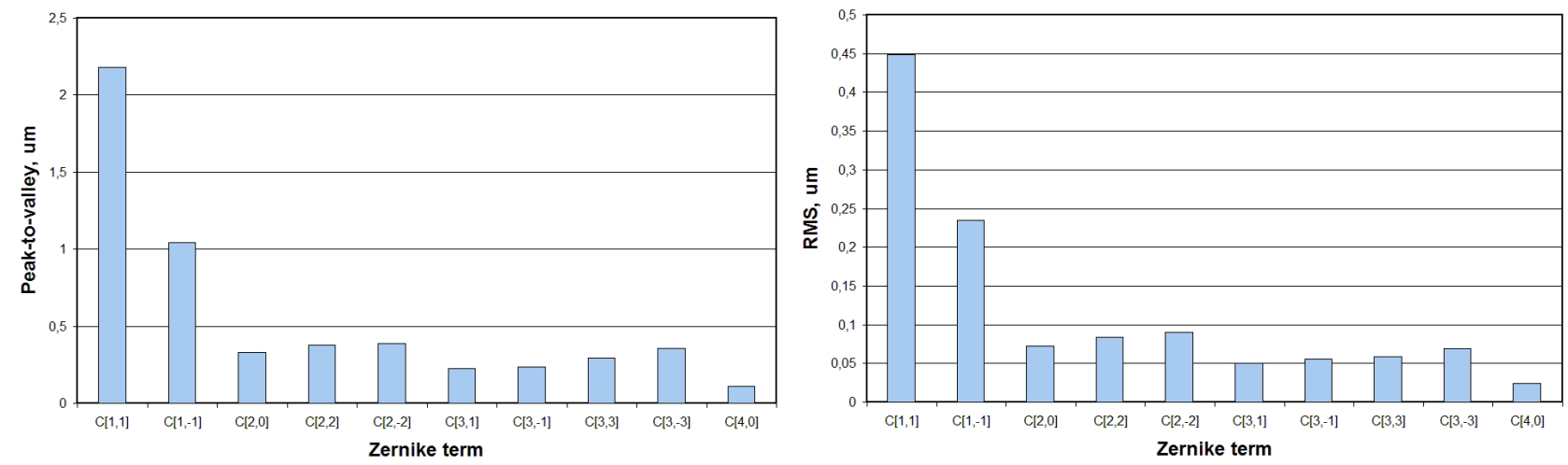

Figure 4. Peak-to-valley and RMS Zernike aberrations measured in a $36 \mathrm{~cm}$ telescope.

As it is seen from Figure 4, only tip and tilt terms contributed into the aberrations considerably; the magnitudes of higher order aberrations were below the diffraction limit. The graphs of tip and tilt are shown in Figure 5. Except for the oscillations caused by the atmospheric turbulence and mechanical vibrations, they contain low-frequency modulation caused by the tracking system error, which provided the most significant contribution. The tracking error consists of

- a linear term, which is caused by inaccurate polar alignment and incorrect motor speed;

- a periodic error, which is caused by imperfections in the mount's worm gear. 

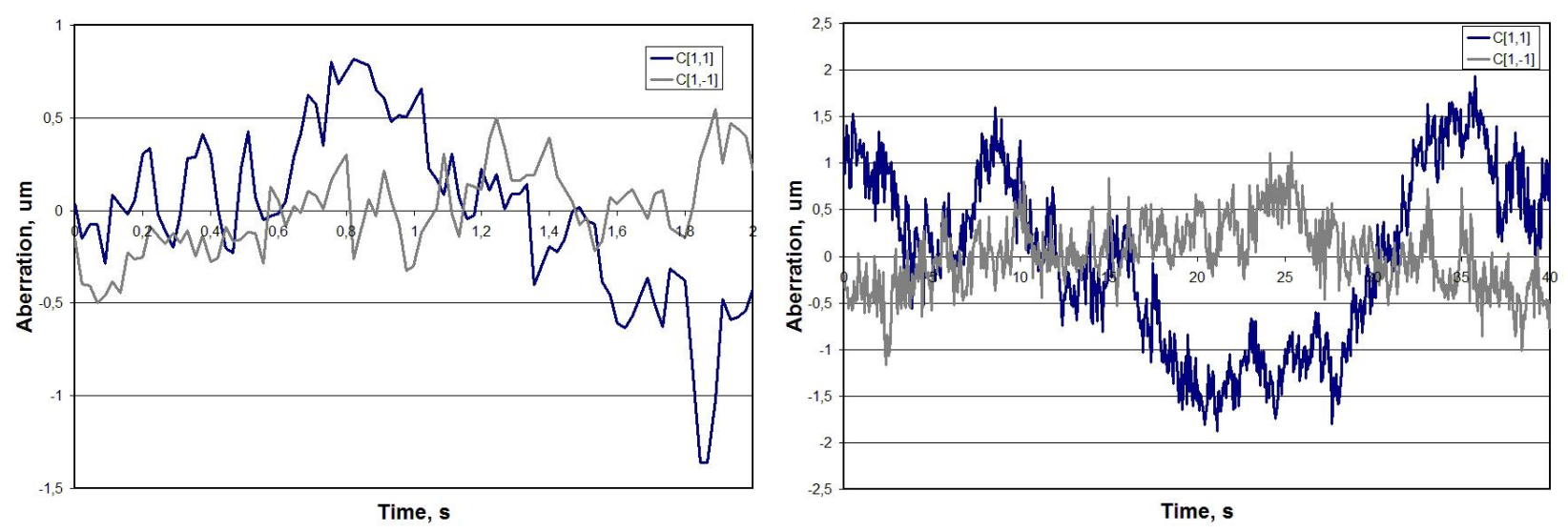

Figure 5. Tip and tilt measured in a $36 \mathrm{~cm}$ telescope; two sample data sets with $2 \mathrm{~s}$ (left) and $40 \mathrm{~s}$ duration (right).
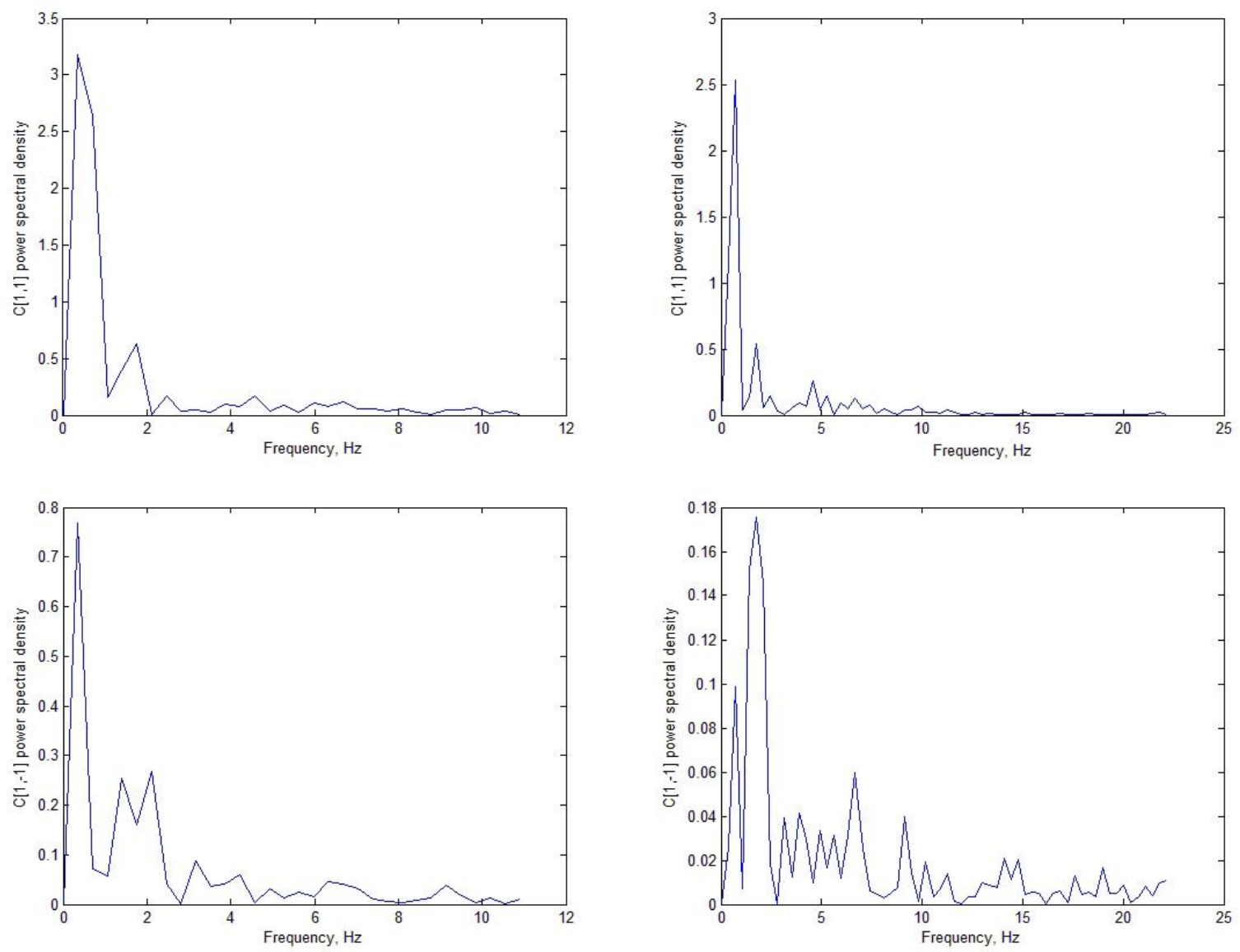

Figure 6. Spectra of tip (top row) and tilt (bottom row) measured in a $36 \mathrm{~cm}$ telescope; calculated from $2 \mathrm{~s}$ unfiltered set of data (left column) and with linear trend extracted (right column). 
Comparing the spectra of the tip and tilt terms (see Figure 6), we can see that the linear drift dominated in term $\mathrm{C}[1,-1]$, and the periodic error in term $\mathrm{C}[1,1]$. In general, the spectra graphs show that correction for low-frequency aberrations below $3 \mathrm{~Hz}$ would greatly improve the system performance.

In another test, we managed to close the loop using Mars both as an object and as a guide star; however, the system did not work stable for longer than $30 \ldots 40$ seconds. It can be explained by the fact that the present system has no dedicated tip-tilt corrector, and the range of tip and tilt correction provided by MMDM was not sufficient to compensate for the tracking error on a relatively long term.

\section{CONCLUSIONS}

We have designed and implemented a compact portable low-cost AO system targeted to a small telescope with a diameter in the range of $25 \mathrm{~cm}$ to $1 \mathrm{~m}$. Laboratory tests have demonstrated satisfactory correction for the statical aberration of the system itself and good correction of the dynamic aberrations introduced by a rotating disk aberrator. The first test on natural sky objects demonstrated stable operation of the AO feedback loop and efficient correction of static aberrations with a natural star of magnitude $\sim 2.21$.

Although the first tests of the system with a natural star were surprisingly successful, more effort is needed to make a product from which the amateur astronomers could benefit. First of all, the existing system is found to be sensitive to the tracking error, which makes the closed-loop feedback unstable. This problem can be solved by incorporation of the tip-tilt corrector in the system design. The range of correction should be sufficiently large to compensate for the tracking error and stabilize the position of the object in the field of view. Currently we are working on implementing this in the next version of the system. It also seems to be attractive to provide an opportunity for interfacing between the control software and telescopes with a computerized tracking system; it would allow largely compensating for the tracking error, keeping it in the safe range.

In fact, in the present tests the system did not demonstrate noticeable image improvement on 25 and $36-\mathrm{cm}$ telescopes because the contribution of the aberrations higher than tip and tilt was too low. However, correction of the atmospheric aberrations makes sense for telescopes with apertures about $1 \mathrm{~m}$ and at altitudes below 45 degrees, where the turbulence effect is much stronger. Besides, a system with similar design can be built for observation of bright moving objects, such as satellites; in this case we deal with faster turbulence dynamics than for stars and planets observation, due to fast object movement. Outside of the astronomic field, the low price and compactness of the system make it very attractive in applications that require low-cost dynamic correction of low-order aberrations, such as laboratory laser setups and vision systems.

\section{ACKNOWLEDGMENTS}

The authors are grateful to Maurice Toet from Sterrenwacht Rijswijk, the Netherlands, for access to a SchmidtCassegrainian telescope and assistance in our experiments. 


\section{REFERENCES}

[1] Discussion in sci.astro.amateur newsgroup: http://groups.google.com/group/sci.astro.amateur/browse_frm/thread/a2e08f4cfb7er768/

[2] Santa Barbara Instrument Group, Inc., AO-8 and AO-L Adaptive Optics systems, http://www.sbig.com/sbwhtmls/ao8.htm

[3] Stellar Products, AO-5 Adaptive Optics system, http://www.stellarproducts.com/adaptive/AO5lit.htm

[4] G. Vdovin and P. M. Sarro, "Flexible mirror micromachined in silicon," Appl. Opt. 34, 2968-2972 (1995).

[5] G. Vdovin, M. Loktev, A. Simonov, V. Kijko, S. Volkov, "Adaptive correction of human-eye aberrations in a subjective feedback loop", Opt. Lett. 30, 795-797 (2005). 